\title{
Cooperation between Polycomb and androgen receptor during oncogenic transformation
}

\author{
Jonathan C. Zhao, ${ }^{1}$ Jianjun $\mathrm{Yu}^{1}{ }^{1}$ Christine Runkle, ${ }^{1}$ Longtao $\mathrm{Wu},{ }^{1}$ Ming Hu, ${ }^{2}$ \\ Dayong Wu, ${ }^{3}$ Jun S. Liu, ${ }^{2}$ Qianben Wang, ${ }^{3}$ Zhaohui S. Qin, ${ }^{4}$ and Jindan $\mathrm{Yu}^{1,5,6}$ \\ ${ }^{1}$ Division of Hematology/Oncology, Department of Medicine, Northwestern University Feinberg School of Medicine, Chicago, Illinois 60611, \\ USA; ${ }^{2}$ Department of Statistics, Harvard University, Cambridge, Massachusetts 02138, USA; ${ }^{3}$ Department of Molecular and Cellular \\ Biochemistry and the Comprehensive Cancer Center, Ohio State University, Columbus, Ohio 43210, USA; ${ }^{4}$ Department of Statistics \\ and Bioinformatics, Emory University, Atlanta, Georgia 30322, USA; ${ }^{5}$ Robert H. Lurie Comprehensive Cancer Center, Northwestern \\ University Feinberg School of Medicine, Chicago, Illinois 60611, USA
}

\begin{abstract}
Androgen receptor (AR) is a hormone-activated transcription factor that plays important roles in prostate development and function, as well as malignant transformation. The downstream pathways of AR, however, are incompletely understood. AR has been primarily known as a transcriptional activator inducing prostate-specific gene expression. Through integrative analysis of genome-wide AR occupancy and androgen-regulated gene expression, here we report AR as a globally acting transcriptional repressor. This repression is mediated by androgen-responsive elements (ARE) and dictated by Polycomb group protein EZH2 and repressive chromatin remodeling. In embryonic stem cells, AR-repressed genes are occupied by EZH2 and harbor bivalent H3K4me3 and H3K27me3 modifications that are characteristic of differentiation regulators, the silencing of which maintains the undifferentiated state. Concordantly, these genes are silenced in castration-resistant prostate cancer rendering a stem cell-like lack of differentiation and tumor progression. Collectively, our data reveal an unexpected role of $A R$ as a transcriptional repressor inhibiting non-prostatic differentiation and, upon excessive signaling, resulting in cancerous dedifferentiation.
\end{abstract}

[Supplemental material is available for this article.]

The transcriptional regulation by androgen receptor (AR), stimulated by androgen, is critical for prostate differentiation and development, as well as malignant transformation. Previous studies have extensively shown that AR induces prostate-specific gene expression driving prostatic differentiation during development (Cunha et al. 2004) and leading to oncogenic transformation during cancer (Heinlein and Chang 2004; Lamont and Tindall 2011). The blockage of AR signaling through androgen deprivation has thus been the mainstay treatment of advanced prostate cancer. While almost all metastatic prostate cancers are initially responsive to androgen ablation therapies, in most cases, however, the disease reemerges in a castration-resistant form. Notably, evidence suggests that this castration-resistant prostate cancer (CRPC) remains dependent on the expression and transcriptional activity of AR through hypersensitive AR activation in the milieu of very low androgen (Chen et al. 2004). The AR pathway therefore remains a leading therapeutic target in CRPC. Recent genomic studies have begun to reveal AR-regulated genes or pathways that might contribute to CRPC.

With the advent of expression microarrays, a large number of genes were found to be regulated by androgen (Wang et al. 2007b). Using chromatin immunoprecipitation (ChIP)-based assays, global AR binding events have been gradually mapped first by regionlimited DNA microarrays (Massie et al. 2007) and later by genometiling arrays (Wang et al. 2009) and ChIP-seq assays (Jia et al. 2008; Lin et al. 2009; Yu et al. 2010b; Massie et al. 2011). These studies have provided unprecedented understanding of AR transcriptional

\section{${ }^{6}$ Corresponding author.}

E-mail jindan-yu@northwestern.edu.

Article published online before print. Article, supplemental material, and publication date are at http://www.genome.org/cgi/doi/10.1101/gr.131508.111. regulation at the genome scale. For example, $>90 \%$ of AR binding events were found at enhancers $>10 \mathrm{~kb}$ away from the transcription start sites (TSS) of coding genes. AR is able to regulate an anabolic transcriptional network to fuel prostate cancer (Massie et al. 2011) and to induce cell cycle genes specifically in CRPC cells (Wang et al. 2009). While a number of these studies observe androgen-repressed genes, these genes and their functions, however, have been somewhat neglected in the favor of androgen-induced genes. Whether or not they represent direct AR target genes and how their repression contributes to prostate cancer are poorly understood.

Previous studies have shown that androgen-repressed genes may also play important roles in prostate cancer cell growth and metastasis (Prescott et al. 2007). Their reexpression during androgen ablation therapy is thought to contribute to disease regression, and they may become repressed once again in CRPC. Despite this importance, only a few studies have reported AR inhibition of a handful of genes (Grosse et al. 2011), a majority of which, however, suggested indirect mechanisms involving inhibition of cofactor proteins with transactivating functions such as SP1 (Verras et al. 2007; Liu et al. 2008; Baniwal et al. 2009; Song et al. 2010). Few of them, indeed, suggested direct AR binding to DNA, however, often through an altered DNA binding specificity (Lanzino et al. 2010; Qi et al. 2011). The evidence to support AR as a transcriptional repressor is lacking, and the exact mechanism largely unknown. Systematic analysis of genomic data will be essential to firmly establish AR as a globally acting transcriptional repressor.

In this study, we present evidence that AR directly inhibits a large number of genes by binding to their regulatory elements containing the consensus ARE motifs. Mechanistically, this repression is mediated by the Polycomb group protein EZH2 and subsequently repressive chromatin remodeling. These genes are developmental regulators functionally involved in cell differentia- 
tion and tumor suppression. Importantly, AR-repressed genes are down-regulated in CRPC cells in an androgen-independent manner. AR-mediated transcriptional repression thus may be an overlooked, yet critical, mechanism to prostate cancer progression and castration resistance.

\section{Results}

\section{AR directly suppresses a large number} of target genes

To identify genes whose expression levels are regulated by androgen, we performed microarray profiling of the androgendependent LNCaP prostate cancer cells treated with $1 \mathrm{nM}$ synthetic androgen $\mathrm{R} 1881$ for $0,3,6,12,24$, and $48 \mathrm{~h}$. The expression of each probe across all time points was normalized to $0 \mathrm{~h}$ to yield fold changes. The probes with greater than threefold change (either up or down) in at least two time points and greater than fourfold change in at least one time point were defined as "androgen-regulated." K-mean clustering followed by heatmap view demonstrated three major clusters: probes gradually induced by androgen $(n=944)$, probes gradually repressed by androgen $(n=939)$, and a small cluster of probes strongly up-regulated at $12 \mathrm{~h}$ but down-regulated at $48 \mathrm{~h}(n=193)$ (Fig. 1A). We defined the first two clusters as "androgen-induced" and "androgenrepressed," corresponding to a unique set of 442 androgen-induced and 428 androgen-repressed RefSeq genes, respectively (Supplemental Table S1). Androgen thus appears to be able to repress as many genes as it induces. To ensure this bidirectional regulation, we further examined these genes in previously published timecourse studies of androgen treatment and confirmed their respective differential regulation by androgen (Supplemental Fig. S1A; Wang et al. 2007a; Massie et al. 2011). We then selected several top repressed genes and confirmed remarkable ( $>10$-fold) down-regulation by androgen in prostate cancer cells (Fig. 1B). In addition, this repression occurred within the physiological range of androgen in a dose-dependent manner (Fig. 1C). To determine whether this androgen repression is mediated by $\mathrm{AR}$, we performed RNA interference of $\mathrm{AR}$ in LNCaP cells. We demonstrate substantial reduction of AR and well-known AR-induced genes such as KLK3 (also known as PSA) and TMPRSS2 upon the removal of AR (Fig. 1D). In contrast, AR knockdown led to remarkable derepression of androgen-repressed genes (Fig. 1E). Interestingly, we found that AR overexpression significantly suppressed the repressed genes even in the absence of androgen (Supplemental Fig. S1B,C). In order to conclude that the repressed genes are directly regulated by $\mathrm{AR}$, however, it is important to determine whether AR binds to their respective regulatory regions.
A
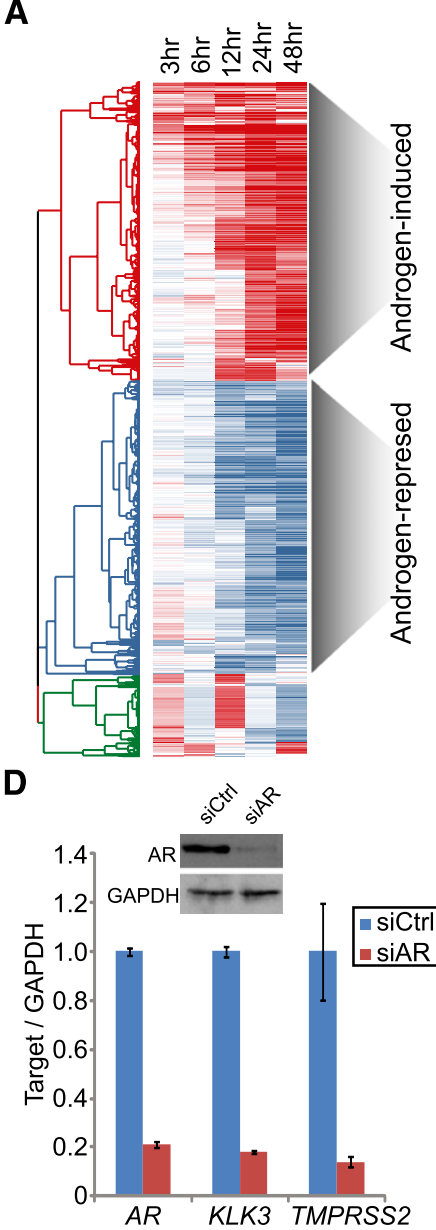

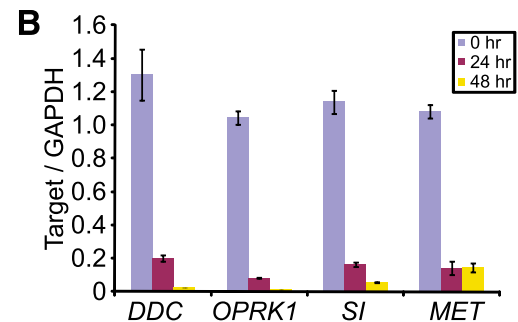

C

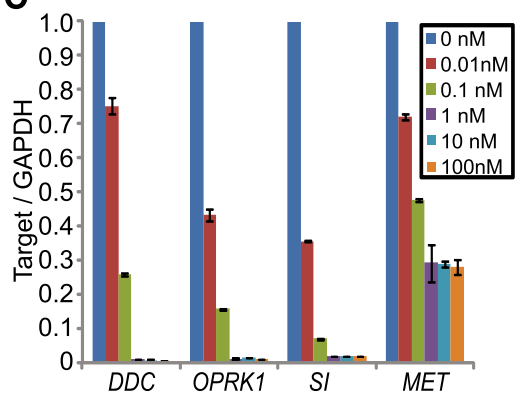

E

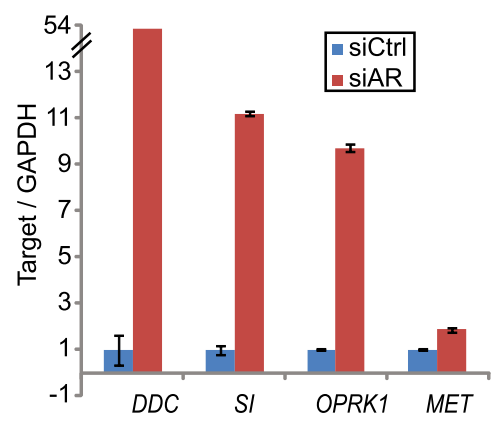

Figure 1. Androgen signaling represses a large set of target genes. $(A)$ Heatmap view of three clusters and treated with $1 \mathrm{nM} \mathrm{R1881}$ for 0, 3, 6, 12, 24, and $48 \mathrm{~h}$. Gene expression was normalized to $0 \mathrm{~h}$. Each five candidate genes. (D) AR knockdown suppresses androgen-induced KLK3 and TMPRSS 2 genes.

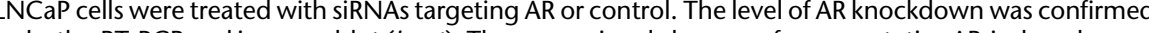
both qRT-PCR and immunoblot (inset). The expressional changes of representative AR-induced genes level of candidate genes in LNCaP cells treated with siRNAs targeting AR or control were measured by qRT-PCR. Error bars: $n=3$, mean \pm SEM. $P<0.01$ relative to control treatment for all experiments.

From the expression microarray data, we noticed that a majority of the androgen-induced and -repressed genes start to show corresponding trends of response at $6 \mathrm{~h}$, which become quite apparent at $12 \mathrm{~h}$ but do not reach peak response until $24 \mathrm{~h}$ after androgen treatment (Fig. 1A). We thus performed ChIP-seq analysis of AR in hormone-deprived LNCaP cells treated with ethanol or androgen for $16 \mathrm{~h}$ to ensure capture of maximum AR binding events (Yu et al. 2010b). We assigned all AR binding events to the nearest genes. We observed that 340 (77\%) androgen-induced and 296 (69\%) of androgen-repressed genes contain at least one AR binding event within their regulatory regions. Because these numbers appeared high relative to other similar studies intersecting binding events and expression changes, we selected a set of androgenunregulated genes that were detected in the microarray but showed no differential regulation by androgen. Out of 445 genes that 
showed $<1.2$-fold differences across all time points, only $185(41 \%)$ contain at least one AR binding event. Overall, AR binding frequency on the repressed genes was only marginally $(P=0.013$, Pearson's $\chi^{2}$ test) lower than the induced genes but was significantly $\left(P=4.52 \times 10^{-16}\right)$ higher than the unregulated genes (Fig. 2A; Supplemental Fig. S2A). To ensure that this is not an observation limited to our data set, we analyzed AR binding on genes induced or repressed by androgen in a previously published data set (Wang et al. 2007a). We found that $>65 \%$ of the genes induced or repressed by androgen contain one AR binding event, which is again significantly $\left(P<0.001\right.$, Pearson's $\chi^{2}$ test $)$ higher than the unregulated genes (Supplemental Fig. S2B). We next examined whether our finding holds true in other independent prostate cancer model systems. Taking a similar approach, we identified genes that are up- or down-regulated by androgen in VCaP cells and analyzed AR binding on these genes using AR ChIP-seq data derived from VCaP cells. Our results confirmed that in VCaP cells there is also significantly more AR binding on androgen-induced $(P<2.2 \times$ $\left.10^{-16}\right)$ and -repressed genes $\left(P=9.4 \times 10^{-7}\right)$ compared with unregulated control genes (Supplemental Fig. S2C). This strongly indicates that, like androgen-induced genes, a majority of the androgen-repressed genes are also directly targeted by AR.
Next, we investigated the distribution of AR binding sites and found that $<5 \%$ of the AR binding events, and to a lesser degree (only $2 \%$ ) for the repressed genes, locate within the promoter regions (between $5 \mathrm{~kb}$ upstream and TSS) of target genes (Fig. 2B). This low number of androgen-repressed genes that contains AR binding sites within the promoters might account for the lack of direct AR-repressed genes reported in the literature. With AR binding events around the repressed genes observed, we next attempted to understand how AR is recruited to the repressed genes. To determine whether any specific DNA motif recruits AR to the target genes, we performed de novo motif search. Our results revealed nearly identical ARE motifs present in the binding sites of both AR-induced and -repressed genes (Fig. 2C). However, AR binding peaks of the induced genes contain significantly $(P<0.001)$ more ARE motifs than the repressed ones, which, in turn, harbor significantly $(P<0.02)$ more than the control regions (Supplemental Fig. S2D). Consistent with these findings, the induced genes contain more strongly enriched AR binding events than the repressed ones (Supplemental Fig. S2E). Therefore, ARE motif appears essential in recruiting AR to both the induced and repressed genes, with relatively more $\mathrm{AR}$ enrichment on the induced genes. We next attempted to confirm individual candidate AR-repressed genes.
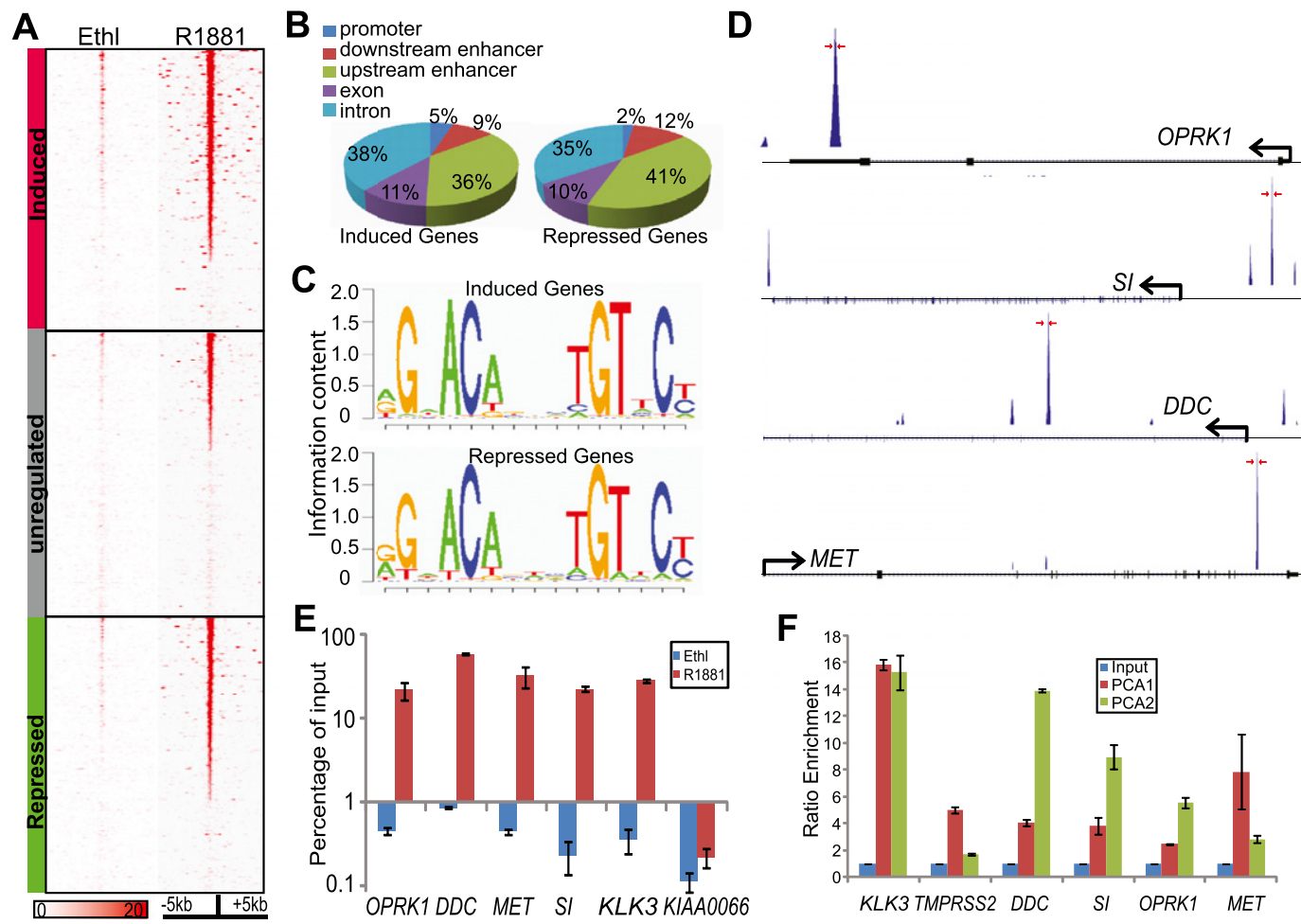

Figure 2. AR directly binds regulatory elements of androgen-repressed genes. ( $A$ ) AR binding density of regions surrounding the highest peak ( $\pm 5 \mathrm{~kb})$ of genes induced, unregulated, or inhibited by androgen. AR ChIP-seq was performed in LNCaP cells treated with ethanol (Ethl) or synthetic androgen R1881 for $16 \mathrm{~h}$. Each row represents one gene, and each column represents the binding intensity in each 100-bp window of the corresponding ChIP-seq experiment. Genes were sorted by the height of their highest AR binding peak in R1881 condition. AR ChIP-seq in Ethl condition was ranked according to R1881 condition. (B) Distribution of AR binding sites relative to AR-induced or AR-repressed genes. AR binding sites within the regulatory regions of ARinduced or -repressed genes were further categorized into promoter (within $5 \mathrm{~kb}$ upstream of the TSS site), enhancer, exon, and intron regions. (C) The most conserved DNA sequence motifs found in the AR binding sites of AR-induced and -repressed genes. De novo motif search was performed using MDscan of the top 500 AR binding sites associated with either AR-induced or -repressed genes. $(D)$ ChIP-seq AR binding peaks on the regulatory elements of candidate AR-repressed genes. Genomic PCR primers (indicated by red arrows) were designed to flank the binding peaks. $(E)$ AR directly binds to the regulatory elements of candidate AR-repressed genes in LNCaP cells. AR ChIP was done in LNCaP cells treated with Ethl or R1881 for $16 \mathrm{~h}$. ChIP-PCR was performed using primers specific to each candidate gene (red arrows in $D), K L K 3$, and a negative control, KIAA0066. Error bars: $n=3$, mean \pm SEM. $(F)$ ChIP-PCR demonstrated AR binding to repressed genes in prostate cancer tissue. AR ChIP was performed in two human prostate cancer tissues. The input and ChIP DNA were first amplified by ligation-mediated PCR, and then an equal amount (50 ng) of the amplicons was used for PCR analysis of target genes. Enrichment in the ChIP DNA was measured relative to the input DNA. Error bars: $n=3$, mean \pm SEM.

\section{Genome Research}

www.genome.org 
Using primers flanking the ChIP-seq AR binding sites (Fig. 2D), we validated AR occupancy on the regulatory elements of selected AR-repressed genes in prostate cancer cells (Fig. 2E). To demonstrate the pathological relevance, we analyzed two human prostate cancer tissues and confirmed AR binding on the repressed genes in primary tissues (Fig. 2F).

\section{AR transcriptional repression is dictated by the Polycomb group protein EZH2}

It is intriguing that AR recruitment to some genes turns on expression, while to others it turns off expression. It has been clearly demonstrated that AR is recruited to the induced genes by FOXA1 and active $\mathrm{H} 3 \mathrm{~K} 4 \mathrm{me} 1$ and $\mathrm{H} 3 \mathrm{~K} 4 \mathrm{me} 2$ histone modifications (Lupien et al. 2008). To determine whether the same mechanisms are involved in AR recruitment to the repressed genes, we analyzed ChIP-seq data of AR, FOXA1, H3K4 methylation, and H3 acetylation. Because AR primarily binds enhancer regions, we examined ChIP-seq read density within $50 \mathrm{~kb}$ upstream to $5 \mathrm{~kb}$ downstream of the TSS of target genes. As previously noted by others (He et al. 2010), H3K4me2 and H3 acetylation were found at both enhancers and promoters, while AR, FOXA1, and $\mathrm{H} 3 \mathrm{~K} 4 \mathrm{me} 1$ predominantly localize at enhancers. Heatmap view of binding densities revealed clear colocalization of AR, FOXA1, H3K4me1, H3K4me2, and H3 acetylation, supporting their collaborative function (Fig. 3A). However, we found that the ChIP-seq read densities of these open chromatin marks are in general substantially stronger for AR-induced than AR-repressed genes.

To further demonstrate how chromatin remodeling and AR recruitment may be altered by androgen, we compared cofactor binding on representative AR-induced and AR-repressed genes before and after androgen stimulation (Fig. 3B). We found that both the induced and repressed genes are pre-marked by precursor modifications even in the absence of androgen. However, these precursor modifications are significantly more enriched on the induced genes with $P$-values of 0.04 and 0.01 for H3K4me1 and H3K4me2, respectively. In contrast, H3 acetylation, a marker of actively transcribed genes, was not significantly $(P=0.11)$ different,
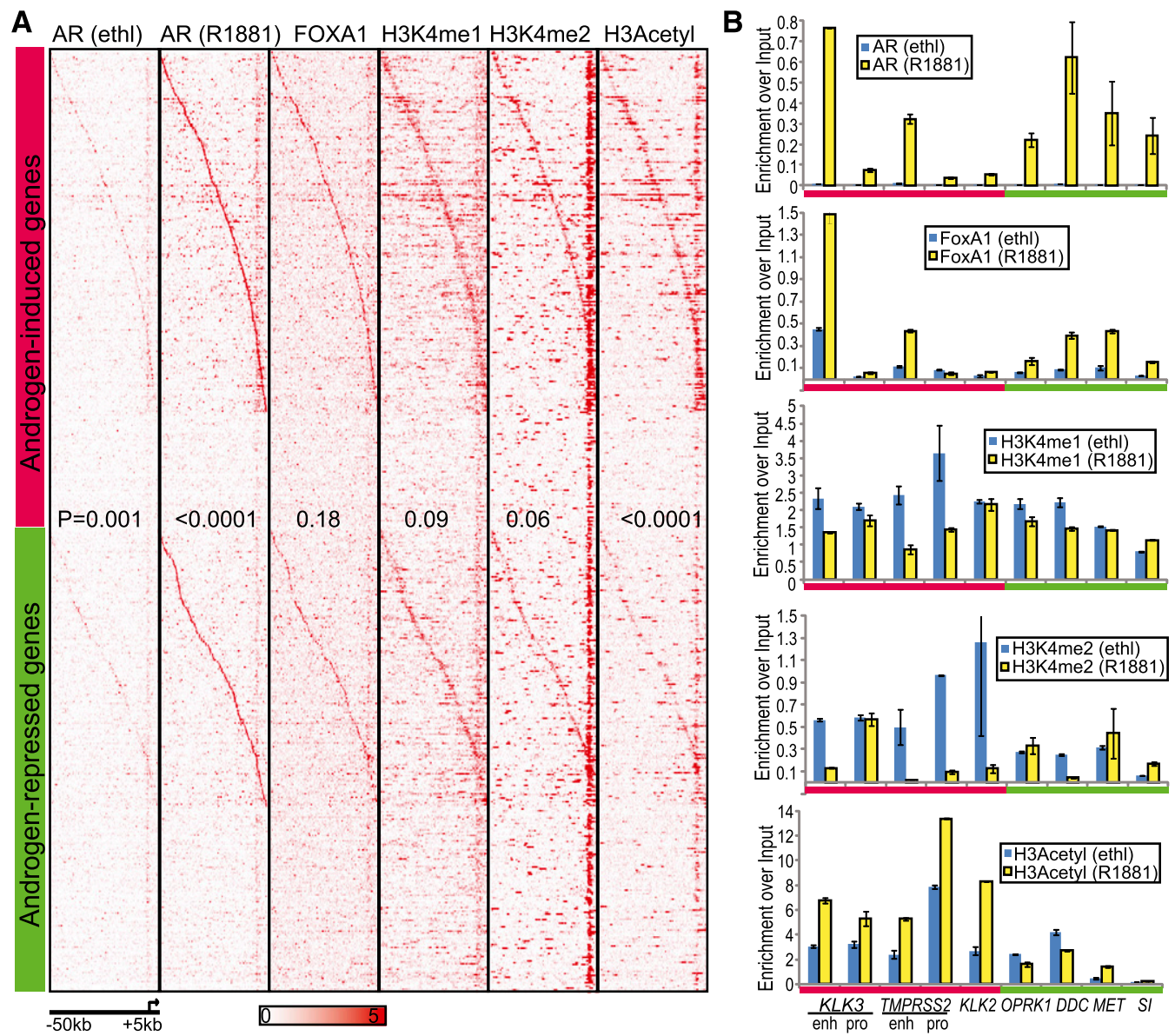

Figure 3. Pioneering factors FOXA1 and H3K4 mono- and di-methylation colocalize with AR at target loci. ( $A$ ) ChIP-seq binding density of regions surrounding the TSS ( $-50 \mathrm{~kb}$ to $+5 \mathrm{~kb}$ ) of AR-induced or -repressed genes. AR ChIP-seq was performed in hormone-starved LNCaP cells treated with ethanol (Ethl) and androgen (R1881). All other ChIP-seq was performed in LNCaP cells grown in regular medium. AR-induced and -repressed genes were each sorted by the distance of the highest AR (R1881) binding peak to the TSS of its corresponding genes; the genes with the highest AR binding peak most upstream of its TSS were ranked on top. Genes that do not contain a peak within -50 to $5 \mathrm{~kb}$ of its TSS were ranked at the bottom of each. The $P$-values indicate differences between the read densities of the two groups of genes. (B) QRT-PCR validation of reduced AR coactivating factor binding on the ARregulated genes. ChIP of AR, FOXA1, H3K4me2, H3K4me2, and Acetyl H3 were performed in hormone-starved LNCaP cells treated with ethanol (Ethl) or androgen (R1881) for $16 \mathrm{~h}$. PCR quantification was carried out using primers flanking the promoters of KLK3 and TMPRSS2 (negative control for AR and FOXA1 binding) and the enhancers of all genes. Marks with significant differences between AR-induced and -repressed genes include H3K4me1 (ethl), H3K4me2 (ethl), and H3 acetyl (R1881) with P-values equal to 0.04, 0.01, and 0.009, respectively. Error bars: $n=3$, mean \pm SEM. 
suggesting that the differences in H3K4me1 and H3K4me2 are unlikely due to the basal expression activities of the AR-induced and AR-repressed genes prior to androgen stimulation. To further confirm this, we analyzed their expression levels in two time-course androgen-treatment studies (Wang et al. 2007a; Massie et al. 2011). Indeed, we observed no significant differences between basal expression levels of induced and repressed genes before AR activation (Supplemental Fig. S3). The expression levels of the induced genes only become significantly higher than the repressed genes after androgen stimulation. Being consistent with the expression status, ChIP-PCR showed that androgen stimulation successfully recruited AR to enhancers of both AR-induced and AR-repressed genes and led to significantly $(P=0.009)$ stronger $\mathrm{H} 3$ acetylation on the induced genes. Collectively, our data suggest that the preexisting $\mathrm{H} 3 \mathrm{~K} 4 \mathrm{me} 1 / 2$ modifications are commonly used to pry open the nucleosomes to allow FOXA1 and AR binding. However, $\mathrm{AR}$ is much more likely to induce genes strongly marked by active histone modifications. With the mechanism of AR recruitment to the repressed genes essentially worked out, we next attempted to understand how AR suppresses gene expression by focusing on the promoter regions.

We examined the ChIP-seq read densities of Pol II, H3K4me3, H3K27me3, and H3K36me3 (Fig. 4A). As expected, AR-induced genes are significantly $(P<0.01)$ enriched for Pol II and H3K4me3 around the TSS and H3K36me3 on the transcribed regions. Intriguingly, H3K27me3 ChIP-seq read density is significantly $(P=$ 0.004) more enriched around AR-repressed genes (Fig. 4A; Supplemental Fig. S4A). Similarly, a higher level of H3K27me3 around AR-repressed genes was also observed in VCaP cells (Supplemental Fig. S4B). By ChIP-PCR, we confirmed stronger enrichment of
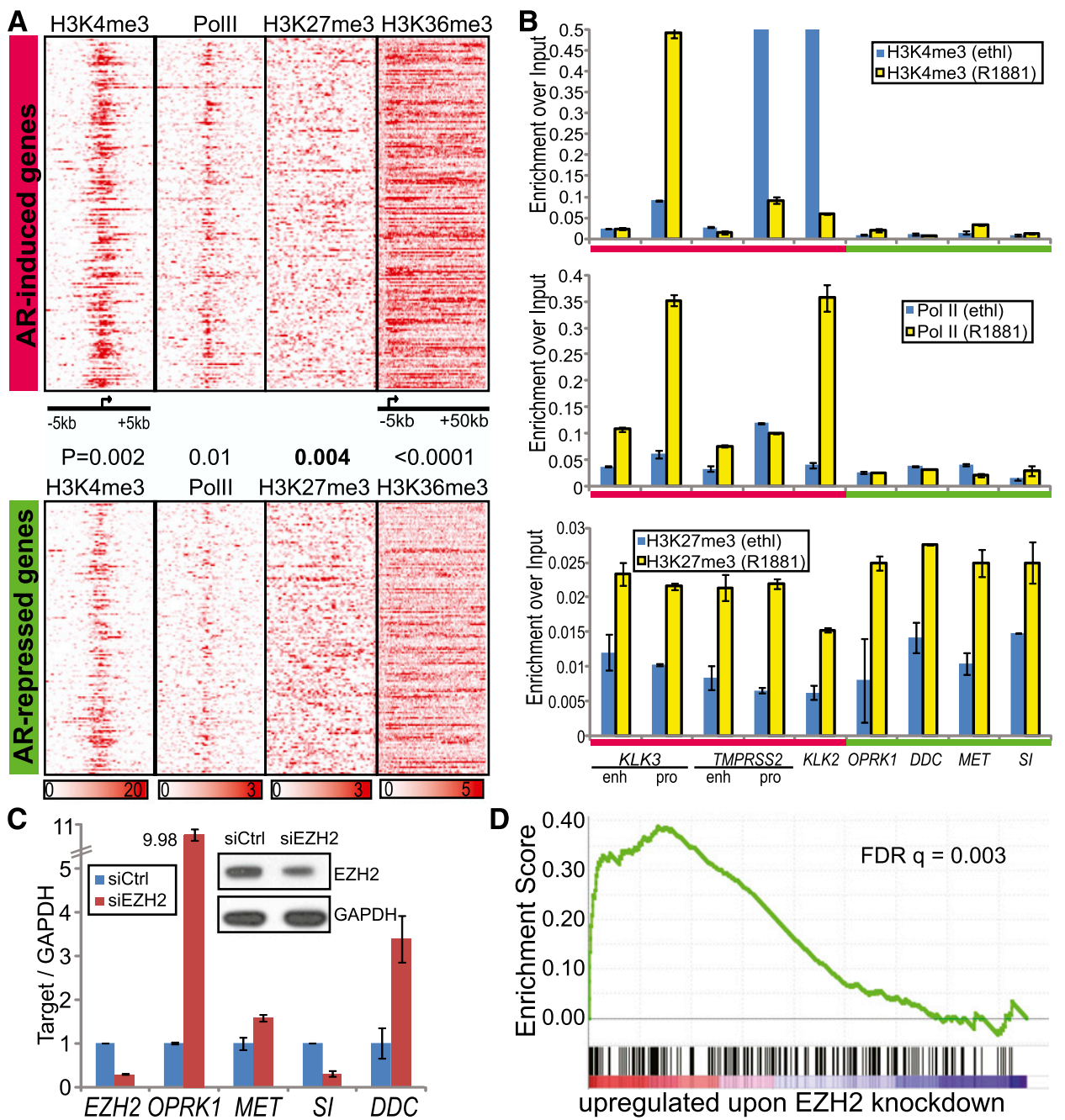

Figure 4. AR-mediated transcriptional repression is dictated by Polycomb group protein EZH2. ( $A$ ) ChIP-seq binding density of regions surrounding the TSS ( $\pm 5 \mathrm{~kb}$ except -5 to $50 \mathrm{~kb}$ for H3k36me3) of AR-induced or -repressed genes. Genes were sorted as in Figure $3 \mathrm{~A}$ except that those not containing a binding peak are not shown. The $P$-values indicate significant differences between the binding densities of AR-induced and -repressed genes. ( $B$ ) Quantitative ChIP-PCR validating AR cofactor binding on AR-regulated genes. ChIP-PCR was performed as described in Figure 3B. Marks with at least marginally significant differences between AR-induced and -repressed genes include Pol II (R1881), H3K27me3 (Ethl), and H3K27me3 (R1881) with Pvalues equal to $0.05,0.09$, and 0.05 , respectively. Error bars: $n=3$, mean \pm SEM. (C) EZH2 knockdown derepresses AR-repressed genes. LNCaP cells were subjected to RNA interference of EZH2 or a non-targeting control. Differential expression of EZH2 and AR-repressed genes was monitored by qRT-PCR. (Inset) Western blot confirming EZH2 knockdown at the protein level. Error bars: $n=3$, mean \pm SEM. (D) GSEA showing enrichment of AR-repressed genes in the genes up-regulated by EZH2 knockdown. LNCaP cells were treated with siEZH2 and subjected to expression profiling. The genes differentially regulated by siEZH 2 were rank ordered and the enrichment of AR-repressed genes assessed.

\section{Genome Research}

www.genome.org 
H3K4me3 and Pol II on the induced gene promoters (Fig. 4B). In contrast, H3K27me3 is significantly $(P=0.05)$ more enriched on the repressed genes, especially after AR activation, supporting repressive chromatin remodeling for gene repression. Because H3K27me3 is enzymatically catalyzed by the Polycomb group protein EZH2, we asked whether EZH2 is involved in AR-mediated gene repression. We carried out ChIP using an anti-EZH2 antibody. Our results demonstrated that EZH2 occupancy on the AR-repressed genes is significantly $(P<0.03)$ increased following AR activation (Supplemental Fig. S4C).

To further examine whether EZH2 is required for AR-mediated transcriptional repression, we performed RNA interference to knock down EZH2 in LNCaP cells (Fig. 4C). Importantly, following $\mathrm{EZH} 2$ knockdown, three out of the four repressed genes tested were markedly derepressed (Fig. 4C). The gene that failed to respond may be regulated by additional repressive mechanisms such as promoter hypermethylation. To confirm the role of EZH2 on a global scale, we performed microarray profiling of these LNCaP cells treated with control and EZH2 siRNA. We identified a cohort of genes that are differentially expressed upon EZH2 knockdown (Supplemental Table S2). Gene Set Enrichment Analysis (GSEA) demonstrated highly significant $(\mathrm{FDR}=0.003)$ up-regulation of AR-repressed genes upon EZH2 knockdown (Fig. 4D). Furthermore, HDAC inhibitor SAHA, which is known to reduce EZH2mediated H3K27me3 (Yu et al. 2007a), derepressed AR-repressed genes alone or in synergy with AR knockdown (Supplemental Fig. $\mathrm{S} 5 \mathrm{~A})$. We then asked whether the derepression of AR-repressed genes is due to the loss of EZH2. We established LNCaP cells with stable EZH2 knockdown using shRNA technology (Supplemental Fig. S5B). Using ChIP followed by PCR, we first verified significant enrichment of H3K27me3 $(P<0.03)$ and EZH2 $(P<0.02)$ on the AR-repressed gene promoters (Supplemental Fig. S5C). We then compared the levels of H3K27me3 and EZH2 in the control cells and the cells with EZH2 knockdown. Our results showed marked reduction of both EZH2 occupancy $(P<0.02)$ and H3K27me3 modification $(P<0.04)$ on the AR-repressed genes upon EZH2 knockdown (Supplemental Fig. S5D,E). Therefore, Polycomb group protein EZH2 appears to collaborate with AR on target gene repression. We next examined whether this regulation is functionally relevant.

\section{AR-repressed genes are developmental regulators suppressed by Polycomb in embryonic stem (ES) cells}

We sought to determine the potential function of the AR-repressed genes by using Molecular Concept Map (MCM) analysis of enrichment by predefined molecular concepts/gene sets in the Oncomine database (http://www.oncomine.com). Out of $\sim 20,000 \mathrm{mo-}$ lecular concepts, only $445(2 \%)$ showed significant $(P<0.001)$ overlap with AR-repressed genes (Supplemental Table S3). As expected, our AR-repressed genes are also found repressed by androgen stimulation in other in vitro and in vivo studies (Fig. $5 \mathrm{~A})$. In addition, many of these genes are down-regulated in aggressive subtypes of a variety of cancers including prostate, breast, and melanoma, suggesting that they may be general tumor-suppressor genes. Importantly, there are significant overlaps between AR-repressed genes and molecular concepts/gene sets relating to Polycomb group (PcG) proteins, such as genes occupied by EZH2, EED, SUZ12, and H3K27me3 in stem cells and genes suppressed in ES cells relative to their differentiated counterparts. Furthermore, MCM analysis showed enrichment of transcription factors such as LEF1, MEIS1, and MEF2 that are involved in development and differentiation. In contrast, AR-induced genes are enriched for prostate-specific gene sets and have no clear correlation with stem cell signatures. These findings pertaining to AR-repressed genes prompted us to further determine whether they may be developmental regulators whose repression maintains undifferentiation in ES cells but contributes to dedifferentiation in cancer.

Characteristic features of developmental regulators in ES cells include PcG protein occupancy and bivalent H3K4me3 and H3K27me3 histone modifications (Lee et al. 2006). We thus obtained a relevant ChIP-seq data set performed in ES cells $(\mathrm{Ku}$ et al. 2008) and examined Polycomb occupancy on the promoters of AR-repressed, AR-induced, and AR-unregulated genes. Not surprisingly, the unregulated genes showed no enriched occupancy by PcG proteins EZH2 and Ring1 (Fig. 5B). Rather, they are strongly occupied by H3K4me3 at the promoters and H3K36me3 at the transcribed regions, supporting their being widely expressed housekeeping genes. Interestingly, some of the AR-induced genes are occupied by PcG and contain bivalent histone modifications, being consistent with their roles in mediating prostatic differentiation. Surprisingly, AR-repressed genes demonstrated the strongest enrichment of PcG occupancy $(P<0.001$, linear mixed effect model) and bivalent H3K4me3 and H3K27me3 $(P<0.001)$ modifications. Therefore, AR-repressed genes appear to be developmental regulators whose expression must be turned off in ES cells to maintain the undifferentiated, pluripotent state. In other words, the expression of these genes in ES cells may lead to cell differentiation, and their loss in differentiated cells is likely to cause dedifferentiation, a hallmark of cancer.

\section{AR-repressed genes remain down-regulated in castration- resistant prostate cancer (CRPC) in the milieu of low androgen}

Since AR remains indispensable for CRPC growth, presumably through regulation of target genes, we next sought to dissect out whether AR-repressed or AR-induced genes or both are accountable for growth of LNCaP-abl, an androgen-independent derivative of LNCaP cells. We hypothesized that if the repressed genes are responsible for CRPC growth, they should be repressed in LNCaP-abl without the need of androgen and vice versa for AR-induced genes. We thus compared the expression of these genes between LNCaP and LNCaP-abl cells, which are regularly maintained in androgendepleted medium. Notably, the repressed genes were expressed in LNCaP-abl at a level similar to LNCaP in the presence of androgen, suggesting their androgen-independent down-regulation in CRPC cells (Fig. 6A). Since AR-repressed genes are likely tumor suppressor genes, their loss may contribute to CRPC cell growth. In contrast, AR-induced genes were barely up-regulated in LNCaP-abl due to the lack of androgen and thus are unlikely to be accountable for androgen-independent LNCaP-abl cell growth (Fig. 6B). However, AR-induced genes may be important for other androgenindependent cells as CRPC is a highly heterogeneous disease. Our data suggest that down-regulation of AR-repressed genes may provide new mechanisms for androgen-independent growth of CRPC cells.

Since AR repression in androgen-responsive cells is facilitated by EZH2, which is often up-regulated in castration-resistant prostate cancer, we next investigated whether the down-regulation of AR-repressed genes in CRPC is due to increased EZH2 regulation. We thus compared the level of Polycomb occupancy on AR-repressed genes between LNCaP-abl and LNCaP cells in the presence and absence of androgen. Concordant with our earlier data, we showed that androgen stimulation of LNCaP cells increases H3K27me3 on the 
A

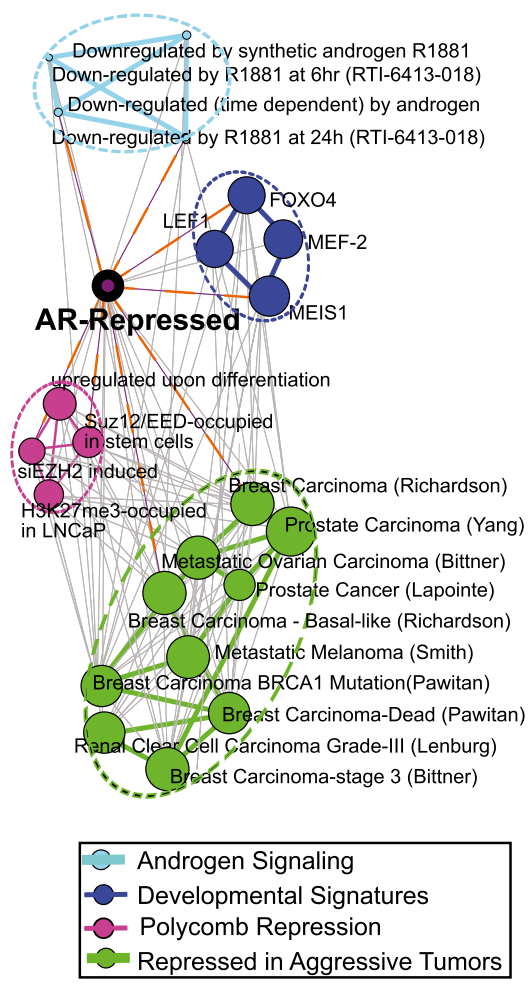

B

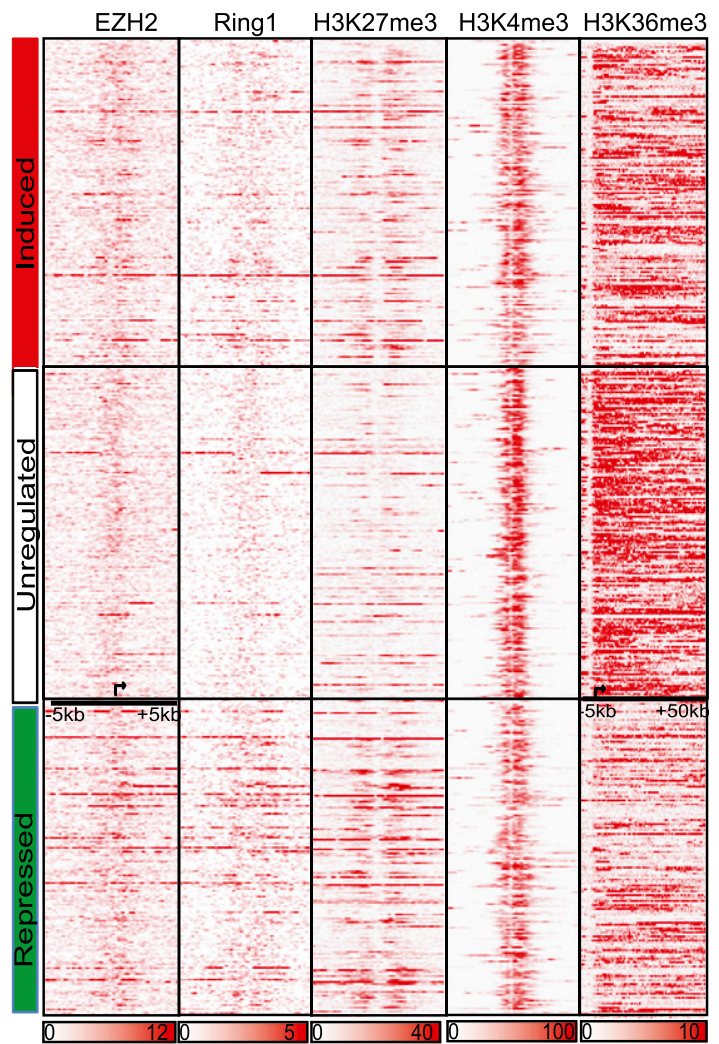

Figure 5. AR-repressed genes are developmental regulators involved in cell differentiation. $(A)$ Network view of the molecular concepts enriched for ARrepressed genes (purple node with black ring). Each node represents a molecular concept or a gene set, with node size proportional to the number of genes within each concept. Each edge represents a statistically significant $\left(P<1 \times 10^{-4}\right)$ (Supplemental Table S3) overlap of genes in the two linked nodes. Enrichments with "androgen signaling" (light blue edges and nodes); enrichments with "developmental signatures" (dark blue); enrichments with "Polycomb repression signatures" (red); and enrichments with "repressed genes in aggressive tumors" (green). (B) ChIP-seq read density of regions surrounding the TSS ( -5 to $50 \mathrm{~kb}$ for H3K36me3 and $\pm 5 \mathrm{~kb}$ for others) of AR-induced or -repressed genes. ChIP-seq data in ES cells were obtained from GSE13084.

repressed genes (Fig. 6C). Remarkably, the level of H3K27me3 modification is significantly stronger in LNCaP-abl compared with LNCaP cells grown in the same androgen-depleted environment. In fact, H3K27me3 level on some genes is even markedly higher than stimulated LNCaP cells. These genes tend to be among the best down-regulated in LNCaP-abl. Our data thus suggest that ARrepressed genes are silenced in CRPC cells in an androgen-independent manner. To confirm this observation on a global scale, we performed microarray analysis of basal gene expression in LNCaP and LNCaP-abl cells in the absence of androgen. GSEA analysis indeed revealed that, while AR-induced genes showed no differential expression, AR-repressed genes are significantly down-regulated in LNCaP-abl cells compared with LNCaP (Fig. 6D).

\section{Discussion}

This is the first study to investigate the regulation and function of $\mathrm{AR}$ as a transcriptional repressor on the global scale. For decades, AR has been primarily considered as a transcriptional activator that induces prostate-specific gene expression. Although androgen stimulation has been shown to also repress genes, these genes are presumed to be indirectly regulated by AR. Little is known about the overall function of AR-repressed genes in prostate cancer. Taking a systems approach, this study establishes AR as a globally acting transcriptional repressor targeting as many genes as it induces. The pioneering factors and ARE motifs used by the AR-induced genes are also involved in recruiting AR to the repressed genes. Once bound to target loci, the repressive function of AR, however, is dictated, at least in part, by the Polycomb group protein EZH2 and consequent H3K27 trimethylation. EZH2 knockdown sufficiently derepresses AR-repressed genes. Furthermore, we provide evidence that, being concordant with the central role of EZH2 in ES cell maintenance, AR-repressed genes are essentially developmental regulators whose expression would otherwise lead to cell differentiation. Our results thus reveal an unexpected role of AR in suppressing cell differentiation (toward non-prostatic lineages). Therefore, AR, previously known as a lineage-specific transcription factor, may play dual roles; it promotes prostatic differentiation via inducing some genes, while simultaneously inhibiting non-prostatic differentiation by suppressing other developmental regulators.

This newly characterized role of AR may be important for normal prostate development and function, as well as malignant transformation. During development, while AR induces prostatespecific gene expression, its suppression of developmental regulators of other lineages is equally important to ensure concomitant silencing of non-prostatic pathways. In mature prostate cells, this newly characterized function of AR may work in concert with the AR-induced genes in stabilizing the prostatic cell type by suppressing potential trans-differentiation. During prostate tumorigenesis,

\section{Genome Research}


A
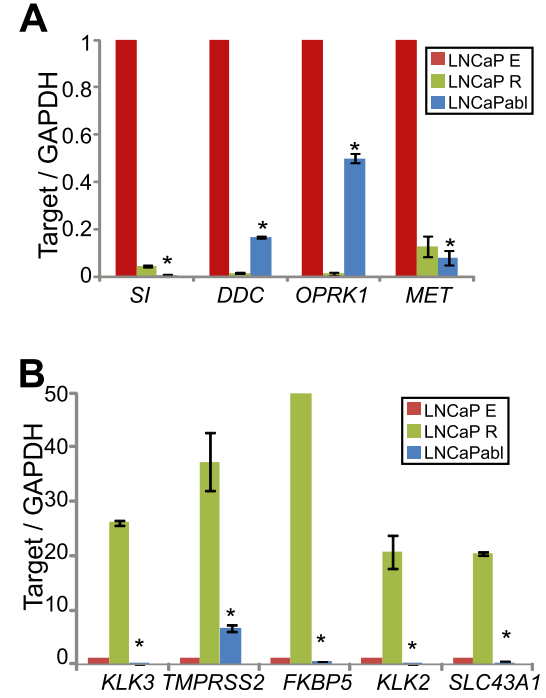

C

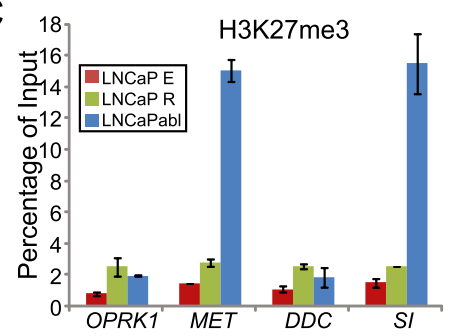

D

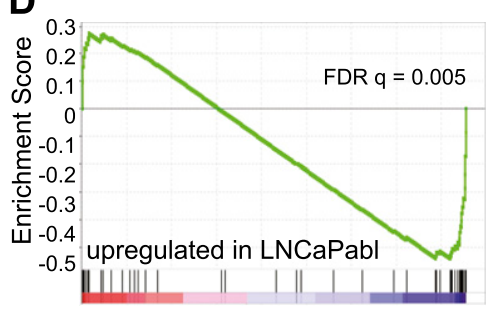

Figure 6. $A R$ repression is maintained by Polycomb in castration-resistant prostate cancer. $(A)$ QRTPCR analysis of AR-repressed and -induced genes in androgen-sensitive LNCaP and castration-resistant LNCaP-abl cells. LNCaP cells were hormone-starved for $72 \mathrm{~h}$ before treatment with ethanol $(E)$ or androgen $(R)$ for $48 \mathrm{~h}$. LNCaP-abl cells were maintained in a hormone-free medium. Target gene expression was first normalized to GAPDH and then to its level at LNCaP treated with ethanol. Error bars: $n=3$, mean \pm SEM. (B) QRT-PCR analysis of AR-induced genes in androgen-sensitive LNCaP and castration-resistant LNCaP-abl cells. (C) H3K27me3 modification on AR-repressed genes. LNCaP cells were hormone-starved for $72 \mathrm{~h}$ and treated with ethanol $(E)$ or androgen $(R)$ for $16 \mathrm{~h}$ before ChIP experiments. LNCaP-abl cells were maintained in a hormone-free medium. Error bars: $n=3$, mean \pm SEM. (D) GSEA showing enrichment of AR-repressed genes with genes down-regulated in LNCaP-abl compared with LNCaP. Gene expression in LNCaP and LNCaP-abl under an androgen-depleted environment was determined. Differentially expressed genes were ranked from up-regulated in LNCaP-abl to downregulated in $\mathrm{LNCaP}$-abl relative to $\mathrm{LNCaP}$.

just like too many AR-induced genes causing aberrant proliferation upon excessive androgen signaling, AR-repressed genes may become overly inhibited rendering cancerous dedifferentiation and/or maldifferentiation. It is, however, important to note that individual genes may have very different, even opposing, functions depending on the disease stage and cellular context. For example, AR-repressed genes that mediate cell differentiation toward certain lineages such as the neuroendocrine phenotype, which has been associated with poor outcome in prostate cancer, may function as oncogenes instead of tumor suppressors. The complexity of prostate cancer biology is further increased by hormone deprivation therapies, which is known to induce neuroendocrine differentiation.

$\mathrm{AR}$ remains required and sufficient to drive CRPC cell growth (Chen et al. 2004). Numerous studies have attempted to understand the molecular mechanisms involved. Most of the findings relate to AR-induced genes/pathways. For example, a recent study reported that, in LNCaP-abl cells, AR acquires new target genes involved in the cell cycle to mediate androgen-independent cell growth (Wang et al. 2009). Our study demonstrates that AR repression of target genes can also contribute to prostate cancer progression through cell dedifferentiation and destabilization. ARrepressed genes remain repressed in LNCaP-abl cells independent of androgen, thus indicating clinical significance of targeting this repressive pathway in a subtype of CRPC tumors.

In this study, we also provide evidence that $\mathrm{EZH} 2$ cooperates with AR in gene repression. Because EZH2 is overexpressed in a majority of aggressive or metastatic prostate cancers, it may continuously suppress AR target genes in an androgen-depleted environment. Our study thus advocates the importance of simultaneously targeting EZH2 as an adjuvant therapy to hormone deprivation in advanced diseases. Interestingly, a recent study has shown that HDAC, a cofactor of EZH2, is also required for AR-induced gene expression (Welsbie et al. 2009). Therefore, inhibitors of HDAC and/or EZH2 may be effective in suppressing both AR-mediated transcriptional activation and repression and thus warrant further investigation. In addition, the levels of key AR-repressed genes may serve as additional biomarkers of prostate cancer and drug efficacy, especially in the cases in which KLK3 failed to respond. Taken together, our study provides innovative revenues for prostate cancer research and may transform the way we perceive and treat prostate cancer.

\section{Methods}

\section{Cell lines and treatments}

The prostate cancer cell lines LNCaP were obtained from ATCC and grown in RPMI supplemented with 10\% FBS (Invitrogen). LNCaP-abl cells were kindly provided by Dr. Qianben Wang (OSU) and were grown in phenol-free RPMI (Invitrogen) supplemented with $10 \%$ charcoal-stripped FCS (Invitrogen). For androgen treatment, cells were hormone-starved for $3 \mathrm{~d}$ before being treated with $1 \mathrm{nM}$ R1881 for $48 \mathrm{~h}$ (for expression assay) or $10 \mathrm{nM}$ R1881 for $16 \mathrm{~h}$ (for ChIP). For RNA interference assays, cells were treated with non-targeting siRNA (D-00110-01, Dharmacon), or siRNAs specific to EZH2 (P-002079-01, Dharmacon) or AR (L-003400, Dharmacon). AR overexpression constructs were a gift from Dr. Stephen Plymate (University of Washington). All transfections were performed using Lipofectamine 2000 (Invitrogen).

\section{ChIP and ChIP-seq assays}

ChIP and ChIP-seq were performed as previously described (Yu et al. 2007a, 2010b). The data set is available at the GEO database as GSE14092. The ChIP antibodies used include AR (\#06-680, Millipore), EZH2 (\#17-662, Millipore), H3K4me3 (ab8580, Abcam), H3K27me3 (\#07-449, Millipore), H3K4me2 (ab7766, Abcam), H3K36me3 (ab9050, Abcam), Acetyl-H3 (ab10812, Abcam), FOXA1 (ab23738, Abcam), SUZ12 (ab12073, Abcam), and RNA Pol II (ab817, Abcam)

\section{ChIP-seq data analysis}

ChIP-seq data were analyzed using HPeak (Qin et al. 2010) to generate window counts and visualized using TreeView. The ChIPseq data set in embryonic stem cells were downloaded from GEO (GSE13084) (Ku et al. 2008). MCM analysis was performed using a query gene list to search for all concepts available in the Oncomine database as previously described (Yu et al. 2007b). Concepts with significant enrichment by the query concept were exported into a supplemental table. Representative concepts were selectively shown as a network in a figure.

The de novo motif search was performed using MDscan (Liu et al. 2002). AR binding sites associated with AR-induced and 
AR-repressed genes were separately ranked by peak height. The sequences of $-200 \mathrm{bp}$ to $+200 \mathrm{bp}$ around the peak summit of the top 500 AR binding sites of either AR-induced or AR-repressed genes were obtained and analyzed by MDscan. The logos were generated using seqLogo in $\mathrm{R}$.

\section{Gene expression microarrays}

Total RNA was extracted using an RNeasy Kit (QIAGEN) and confirmed for RNA integrity. Microarray analysis was performed using HumanHT-12 v 4.0 Expression BeadChip (Illumina). The beadlevel data were preprocessed using GenomeStudio (Illumina), and the expression data were analyzed using the beadarray package in Bioconductor, $\log _{2}$-transformed, and quantile-normalized using the beadarray package. Enrichment of gene sets was analyzed using the Gene Set Enrichment Analysis (GSEA) tool (Broad Institute). All gene expression data have been uploaded to the GEO database.

\section{Quantitative PCR}

QRT-PCR or quantitative ChIP-PCR was performed using GoTaq qPCR Master Mix $2 \times$ (Promega) on an Applied Biosystems StepOne Plus Real Time PCR System as previously described (Yu et al. 2007b, 2010a).

\section{Western blotting}

After washing twice with cold PBS, cells were lysed and sonicated briefly in NP-40 buffer (Invitrogen) containing protease inhibitors (Roche Applied Science). Frozen tissue samples were homogenized in NP-40 buffer containing protease inhibitors. All lysates were centrifuged to remove cellular debris, and the supernatants were used for Western blotting. The antibodies used for Western blotting were anti-GAPDH (monoclonal, Abcam), anti-AR (polyclonal PG21, Millipore), and anti-EZH2 (BD).

\section{Data access}

New high-throughput data generated in this study have been submitted to the NCBI Gene Expression Omnibus (GEO) (http:// www.ncbi.nlm.nih.gov/geo/) under accession number GSE31978.

\section{Acknowledgments}

We thank Dr. Stephen Plymate for the AR overexpression constructs and Drs. Timothy Kuzel, Chung Lee, Raymond Bergan, and Robin Leikin for helpful discussions. We are grateful to Dr. Arul M. Chinnaiyan for his support. This work was supported by the NIH P50CA69568 Career Development Award (to J.Y.), U54CA143869 pilot project (to J.Y.), R00CA129565 (to J.Y.), R01CA151979 (to Q.W.), and R01HG005119 (to Z.Q.), the U.S. Department of Defense PC080665 (to J.Y.), and the American Cancer Society Research Scholar Award RSG-12-085-01 (to J.Y.).

\section{References}

Baniwal SK, Khalid O, Sir D, Buchanan G, Coetzee GA, Frenkel B. 2009. Repression of Runx2 by androgen receptor (AR) in osteoblasts and prostate cancer cells: AR binds Runx2 and abrogates its recruitment to DNA. Mol Endocrinol 23: 1203-1214.

Chen CD, Welsbie DS, Tran C, Baek SH, Chen R, Vessella R, Rosenfeld MG Sawyers CL. 2004. Molecular determinants of resistance to antiandrogen therapy. Nat Med 10: 33-39.

Cunha GR, Ricke W, Thomson A, Marker PC, Risbridger G, Hayward SW, Wang YZ, Donjacour AA, Kurita T. 2004. Hormonal, cellular, and molecular regulation of normal and neoplastic prostatic development. I Steroid Biochem Mol Biol 92: 221-236.
Grosse A, Bartsch S, Baniahmad A. 2011. Androgen receptor-mediated gene repression. Mol Cell Endocrinol doi: 10.1016/j.mce.2011.06.032.

He HH, Meyer CA, Shin $\mathrm{H}$, Bailey ST, Wei G, Wang Q, Zhang Y, Xu K, Ni M, Lupien M, et al. 2010. Nucleosome dynamics define transcriptional enhancers. Nat Genet 42: 343-347.

Heinlein CA, Chang C. 2004. Androgen receptor in prostate cancer. Endocr Rev 25: 276-308.

Jia L, Berman BP, Jariwala U, Yan X, Cogan JP, Walters A, Chen T, Buchanan G, Frenkel B, Coetzee GA. 2008. Genomic androgen receptor-occupied regions with different functions, defined by histone acetylation, coregulators and transcriptional capacity. PLOS ONE 3: e3645. doi: 10.1371 /journal.pone.0003645

Ku M, Koche RP, Rheinbay E, Mendenhall EM, Endoh M, Mikkelsen TS Presser A, Nusbaum C, Xie X, Chi AS, et al. 2008. Genomewide analysis of PRC1 and PRC2 occupancy identifies two classes of bivalent domains. PLoS Genet 4: e1000242. doi: 10.1371/journal.pgen.1000242.

Lamont KR, Tindall DJ. 2011. Minireview: Alternative activation pathways for the androgen receptor in prostate cancer. Mol Endocrinol 25: 897907.

Lanzino M, Sisci D, Morelli C, Garofalo C, Catalano S, Casaburi I, Capparelli C, Giordano C, Giordano F, Maggiolini M, et al. 2010. Inhibition of cyclin D1 expression by androgen receptor in breast cancer cellsidentification of a novel androgen response element. Nucleic Acids Res 38: $5351-5365$

Lee TI, Jenner RG, Boyer LA, Guenther MG, Levine SS, Kumar RM, Chevalier $\mathrm{B}$, Johnstone SE, Cole MF, Isono K, et al. 2006. Control of developmental regulators by Polycomb in human embryonic stem cells. Cell 125: 301313

Lin B, Wang J, Hong X, Yan X, Hwang D, Cho JH, Yi D, Utleg AG, Fang X, Schones DE, et al. 2009. Integrated expression profiling and ChIP-seq analyses of the growth inhibition response program of the androgen receptor. PLOS ONE 4: e6589. doi: 10.1371/journal pone.0006589.

Liu XS, Brutlag DL, Liu JS. 2002. An algorithm for finding protein-DNA binding sites with applications to chromatin-immunoprecipitation microarray experiments. Nat Biotechnol 20: 835-839.

Liu YN, Liu Y, Lee HJ, Hsu YH, Chen JH. 2008. Activated androgen receptor downregulates E-cadherin gene expression and promotes tumor metastasis. Mol Cell Biol 28: 7096-7108.

Lupien M, Eeckhoute J, Meyer CA, Wang Q, Zhang Y, Li W, Carroll JS, Liu XS, Brown M. 2008. FoxA1 translates epigenetic signatures into enhancerdriven lineage-specific transcription. Cell 132: 958-970.

Massie CE, Adryan B, Barbosa-Morais NL, Lynch AG, Tran MG, Neal DE, Mills IG. 2007. New androgen receptor genomic targets show an interaction with the ETS1 transcription factor. EMBO Rep 8: 871-878.

Massie CE, Lynch A, Ramos-Montoya A, Boren J, Stark R, Fazli L, Warren A, Scott H, Madhu B, Sharma N, et al. 2011. The androgen receptor fuels prostate cancer by regulating central metabolism and biosynthesis. EMBO J 30: 2719-2733

Prescott J, Jariwala U, Jia L, Cogan JP, Barski A, Pregizer S, Shen HC, Arasheben A, Neilson JJ, Frenkel B, et al. 2007. Androgen receptormediated repression of novel target genes. Prostate 67: 1371-1383.

Qi W, Gao S, Chu J, Zhou L, Wang Z. 2011. Negative androgen-response elements mediate androgen-dependent transcriptional inhibition of $T G F-\beta 1$ and CDK2 promoters in the prostate gland. J Androl doi: 10.2164/jandrol.110.011999.

Qin ZS, Yu J, Shen J, Maher CA, Hu M, Kalyana-Sundaram S, Chinnaiyan AM. 2010. HPeak: An HMM-based algorithm for defining read-enriched regions in ChIP-Seq data. BMC Bioinformatics 11: 369. doi: 10.1186/ 1471-2105-11-369.

Song K, Wang H, Krebs TL, Wang B, Kelley TJ, Danielpour D. 2010. DHT selectively reverses Smad3-mediated/TGF- $\beta$-induced responses through transcriptional down-regulation of Smad3 in prostate epithelial cells Mol Endocrinol 24: 2019-2029.

Verras M, Lee J, Xue H, Li TH, Wang Y, Sun Z. 2007. The androgen receptor negatively regulates the expression of c-Met: Implications for a novel mechanism of prostate cancer progression. Cancer Res 67: 967-975.

Wang Q, Li W, Liu XS, Carroll JS, Janne OA, Keeton EK, Chinnaiyan AM, Pienta KJ, Brown M. 2007a. A hierarchical network of transcription factors governs androgen receptor-dependent prostate cancer growth. Mol Cell 27: 380-392.

Wang XD, Wang BE, Soriano R, Zha J, Zhang Z, Modrusan Z, Cunha GR, Gao WQ. 2007b. Expression profiling of the mouse prostate after castration and hormone replacement: Implication of $\mathrm{H}$-cadherin in prostate tumorigenesis. Differentiation 75: 219-234.

Wang Q, Li W, Zhang Y, Yuan X, Xu K, Yu J, Chen Z, Beroukhim R, Wang H, Lupien $M$, et al. 2009. Androgen receptor regulates a distinct transcription program in androgen-independent prostate cancer. Cell 138: $245-256$.

Welsbie DS, Xu J, Chen Y, Borsu L, Scher HI, Rosen N, Sawyers CL. 2009 Histone deacetylases are required for androgen receptor function in 


\section{AR directly inhibits non-prostatic differentiation}

hormone-sensitive and castrate-resistant prostate cancer. Cancer Res 69: 958-966.

Yu J, Cao Q, Mehra R, Laxman B, Yu J, Tomlins SA, Creighton CJ, Dhanasekaran SM, Shen R, Chen G, et al. 2007a. Integrative genomics analysis reveals silencing of $\beta$-adrenergic signaling by Polycomb in prostate cancer. Cancer Cell 12: 419-431.

Yu J, Rhodes DR, Tomlins SA, Cao X, Chen G, Mehra R, Wang X, Ghosh D, Shah RB, Varambally S, et al. 2007b. A Polycomb repression signature in metastatic prostate cancer predicts cancer outcome. Cancer Res 67: 10657-10663.
Yu J, Cao Q, Yu J, Wu L, Dallol A, Li J, Latif F, Wu J, Chinnaiyan AM. 2010a. The neuronal repellent SLIT2 is a target for repression by EZH2 in prostate cancer. Oncogene 29: 5370-5380.

Yu J, Mani RS, Cao Q, Brenner CJ, Cao X, Wang X, Wu L, Li J, Hu M, Gong Y, et al. 2010b. An integrated network of androgen receptor, Polycomb, and TMPRSS2-ERG gene fusions in prostate cancer progression. Cancer Cell 17: 443-454.

Received September 2, 2011; accepted in revised form November 29, 2011. 


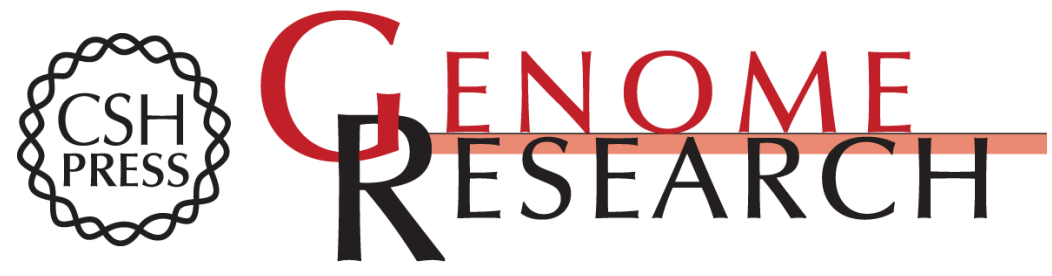

\section{Cooperation between Polycomb and androgen receptor during oncogenic transformation}

Jonathan C. Zhao, Jianjun Yu, Christine Runkle, et al.

Genome Res. 2012 22: 322-331 originally published online December 16, 2011

Access the most recent version at doi:10.1101/gr.131508.111

Supplemental Material

References

License

Email Alerting Service
http://genome.cshlp.org/content/suppl/2011/12/16/gr.131508.111.DC1

This article cites 28 articles, 4 of which can be accessed free at: http://genome.cshlp.org/content/22/2/322.full.html\#ref-list-1

Receive free email alerts when new articles cite this article - sign up in the box at the top right corner of the article or click here.

\section{Affordable, Accurate Sequencing.}

To subscribe to Genome Research go to:

https://genome.cshlp.org/subscriptions 